\title{
Rationale and design of ASPIRE-ICU: a prospective cohort study on the incidence and predictors of Staphylococcus aureus and Pseudomonas aeruginosa pneumonia in the ICU
}

Fleur P. Paling ${ }^{1 *} \mathbb{D}$, Darren P. R. Troeman ${ }^{1}$, Martin Wolkewitz ${ }^{2}$, Rubana Kalyani ${ }^{3}$, Daniël R. Prins ${ }^{1}$, Susanne Weber ${ }^{2}$, Christine Lammens ${ }^{4}$, Leen Timbermont ${ }^{4}$, Herman Goossens ${ }^{4}$, Surbhi Malhotra-Kumar ${ }^{4}$, Frangiscos Sifakis ${ }^{5}$, Marc J. M. Bonten ${ }^{1,6}$ and Jan A. J. W. Kluytmans $s^{1,7}$

\begin{abstract}
Background: The epidemiology of ICU pneumonia caused by Staphylococcus aureus (S. aureus) and Pseudomonas aeruginosa (P. aeruginosa) is not fully described, but is urgently needed to support the development of effective interventions. The objective of this study is to estimate the incidence of $S$. aureus and $P$. aeruginosa ICU pneumonia and to assess its association with patient-related and contextual risk factors.

Methods: ASPIRE-ICU is a prospective, observational, multi-center cohort study nested within routine surveillance among ICU patients in Europe describing the occurrence of S. aureus and P. aeruginosa ICU pneumonia. Two thousand (2000) study cohort subjects will be enrolled (50\% S. aureus colonized) in which specimens and data will be collected. Study cohort subjects will be enrolled from a larger surveillance population, in which basic surveillance data is captured. The primary outcomes are the incidence of $S$. aureus ICU acquired pneumonia and the incidence of $P$. aeruginosa ICU acquired pneumonia through ICU stay.

The analysis will include advanced survival techniques (competing risks and multistate models) for each event separately as well as for the sub-distribution of ICU pneumonia to determine independent association of outcomes with risk factors. A risk prediction model will be developed to quantify the risk for acquiring $S$. aureus or $P$. aeruginosa ICU pneumonia during ICU stay by using a composite score of independent risk factors.
\end{abstract}

Discussion: The diagnosis of pathogen-specific ICU pneumonia is difficult, however, the criteria used in this study are objective and comparable to those in the literature.

Trial registration: This study is registered on clinicaltrials.gov under identifier NCT02413242.

Keywords: ICU pneumonia, S. aureus, P. aeruginosa, Vap

\footnotetext{
* Correspondence: f.p.paling@umcutrecht.nl

1 Julius Center for Health Sciences and Primary Care, University Medical

Center Utrecht, HP Stratenum 6.131, PO Box 85500, 3508 GA Utrecht, The

Netherlands

Full list of author information is available at the end of the article
} 


\section{Background}

Patients hospitalized in the Intensive Care Unit (ICU) are at risk of acquiring pneumonia, especially if they are mechanically ventilated. Despite extensive efforts, ICU-acquired pneumonia continues to be one of the most frequently occurring complications in the ICU and increases morbidity as well as mortality $[1,2]$. Accurately describing and predicting the occurrence of ICU pneumonia is difficult as a result of inconsistencies in the case definition and surveillance methods in different countries $[1,3]$. The need for an accurate and standardized prognosis is important considering current interventions are preventive rather than therapeutic [4]. As part of the COMBACTE consortium (COMBatting AntibiotiC resisTance in Europe), two monoclonal antibodies (mAbs) are currently being developed that target $S$. aureus and $P$. aeruginosa, respectively $[5,6]$. Both bacteria are frequently occurring causative pathogens of ICU pneumonia and administration of a mAb may prevent the development of ICU pneumonia with these pathogens $[7,8]$. Prospective studies can assess possible risk factors that can identify subsets of patients that may benefit most from these interventions. Thus, the goal of this study is to systematically assess the impact of patient-related and contextual factors on the incidence of $S$. aureus and $P$. aeruginosa ICU pneumonia in Europe and to identify the patient subgroups that are at greater risk for disease and bear a disproportionate disease burden. These objectives will directly contribute to the sample size and feasibility calculations for clinical trial design of the mAb interventions [8].

\section{Methods}

\section{Objectives}

The primary objectives of this study are to determine the incidence of:

(1)S. aureus ICU pneumonia through ICU stay; and

(2)P. aeruginosa ICU pneumonia through ICU stay; and their independent associations with patient-related factors (e.g. colonization status, baseline serum antibody levels against $S$. aureus or $P$. aeruginosa antigens) and contextual factors.

The key secondary objectives are to develop risk prediction models to quantify the risk of acquiring (i) $S$. aureus or (ii) $P$. aeruginosa ICU pneumonia during ICU stay, by using a composite score of independent risk factors identified through primary objective 1 and 2 . Other secondary and exploratory objectives can be found on ClinicalTrials.gov, under identifier NCT02413242, or in the online (Additional file 1: S.01).

\section{Study design}

ASPIRE-ICU (Advanced understanding of Staphylococcus aureus and Pseudomonas aeruginosa Infections in
EuRopE - Intensive Care Units) is a multi-center, prospective, observational cohort study nested within ongoing routine surveillance among ICU patients in Europe. The study is composed of two study populations, the surveillance population and study cohort population. The study cohort is nested within the larger surveillance population; this means that all data and specimens collected specifically for study cohort participants is in addition to data already captured by ways of surveillance. An overview of the schedule of procedures, including all sample collection types and time points can be found in the online (Additional file 1: Table S.02).

\section{Study populations and recruitment Surveillance population}

Patients eligible to participate in the surveillance population must be on mechanical ventilation (MV) upon or (expected to be) within $24 \mathrm{~h}$ after ICU admission and have an expected length of stay (LOS) of at least $48 \mathrm{~h}$. Patients with an expected ICU stay of less than $48 \mathrm{~h}$ are at a lower risk for developing ICU infections since this population is generally healthier, without significant comorbidities and shorter in the ICU.

The surveillance population are considered to be the source population from which the study cohort subjects are derived. No informed consent is required for participation in the surveillance population, but depending on local legislation, patients of the surveillance population will receive information (i.e. leaflet/flyer) on the ASPIRE-ICU study and are able to deny use of their de-identified data for scientific purposes.

\section{Study cohort population}

Surveillance patients that meet the eligibility criteria described below for the study cohort population will be enrolled. 2000 study cohort subjects are required to meet the objectives of this study. Study cohort subjects are approached for informed consent based on their S. aureus colonization status at ICU admission. Subjects will be enrolled in a 1:1 ratio of $S$. aureus colonized subjects to non $S$. aureus colonized subjects with 1000 subjects in each stratum. A similar temporal distribution of enrolled $S$. aureus colonized and non S. aureus -colonized subjects will be managed by selecting the first non-colonized subject after including a colonized subject. For subjects unable to provide consent for any reason, a legally accepted representative may consent on the subject's behalf at the time of enrollment.

Inclusion criteria for study cohort

1. Participant is 18 years or older at the time of enrollment. 
2. Participant is on mechanical ventilation at ICU admission, or is (expected to be) within $24 \mathrm{~h}$ thereafter, based on investigator's judgment.

3. Expected stay in ICU is $48 \mathrm{~h}$ or longer based on investigator's judgment.

4. S. aureus colonization status is known within $72 \mathrm{~h}$ after start of first episode of mechanical ventilation and according to the result, the patient qualifies for enrollment.

5. Written informed consent from subject / legally accepted representative within $72 \mathrm{~h}$ after start of first episode of mechanical ventilation.

Exclusion criteria for study cohort

1. Previous participation as a subject in the study cohort of this study.

2. Simultaneous participation of the subject in any preventive experimental study into antistaphylococcus or anti-pseudomonas aeruginosa interventions.

3. Expected death (moribund status) within $48 \mathrm{~h}$, or ICU discharge of the participant within $24 \mathrm{~h}$, at the moment of informed consent.

\section{Study outcome definitions}

Considering that the definition of our primary outcome, ICU pneumonia caused by $S$. aureus or $P$. aeruginosa, is very extensive, we would like to refer to the (Additional file 1: S.03) containing the full definition. In summary, ICU pneumonia is defined as pneumonia occurring $\geq 48 \mathrm{~h}$ after admission to the ICU and is confirmed by a new or worsening infiltrate on chest X-ray or CT-thorax. Furthermore, the patient must fulfill specific clinical criteria (for example abnormal temperature, production of sputum, auscultatory abnormalities, acute changes in the ventilatory support system), in addition to at least 1 microbiological criterion (positive respiratory specimen, blood culture, pleural fluid aspirate or lung tissue culture).

\section{Site selection}

In total, 30 sites in 11 countries were selected from sources including but not limited to the COMBACTE CLIN-Net and LAB-Net databases [7]. To ensure the pan-European continent is represented, there is at least one country included from the Northern / Southern / Eastern and Western region of Europe. The selected sites adequately balance different factors such as geography, background antibiotic resistance prevalence, etc.. To ensure enrollment is met within the expected timelines, back-up sites were selected for various reasons (i.e. low enrollment numbers, decline further participation) that can supplement or replace primary sites selected. For each site also a local laboratory was selected to participate in the study.

A Site Selection Committee selected sites and laboratories based on pre-defined criteria in the Site Selection Plan. Assessment of these criteria was aided by site feasibility questionnaires. Participating ICUs must have a routine $S$. aureus screening protocol in order to be selected for participation.

Screening should consist of a minimum of one nasal swab and one lower respiratory tract (LRT) sample analyzed locally on the day of ICU admission. The LRT sample is defined as the collection of an endotracheal aspirate (ETA) sample, or, if an ETA cannot be collected, a sputum sample may be taken. As an exception, for routine $S$. aureus colonization screening at ICU admission only, if both the ETA and sputum cannot be collected, a throat swab may be taken.

\section{Statistical analysis Sample size calculation}

Sample size calculations are based on the expected incidence precision of $S$. aureus ICU pneumonia and $P$. aeruginosa ICU pneumonia, since the primary objective of the study is to identify the patient groups most at risk for this outcome.

Assuming an incidence of $S$. aureus ICU pneumonia of $12.5 \%$ and $1.5 \%$ in the $S$. aureus colonized group and non $S$. aureus colonized group respectively, this would result in an overall incidence of $7 \%$ within the 2000 study cohort subjects, or $140 \mathrm{~S}$. aureus ICU pneumonia endpoints [9]. The overall incidence estimate would have precision of $1.12 \%$ (95\% confidence interval [CI]: $5.9 \%-$ 8.12\%, using normal approximation).

For $P$. aeruginosa ICU pneumonia, the overall incidence is estimated regardless of colonization status at ICU admission. Assuming an overall incidence of $P$. aeruginosa ICU pneumonia of $2.5 \%$ within the 2000 study cohort subjects, this would result in $50 \mathrm{P}$. aeruginosa ICU pneumonia endpoints. The overall incidence estimate would have precision of $0.68 \%$ (95\% CI $1.82 \%-3.18 \%$, using normal approximation).

\section{Planned analysis}

The primary analysis will evaluate the incidence density of $S$. aureus or $P$. aeruginosa ICU pneumonia; its calculation will depend on time from admission (for outcome ICU pneumonia) or time from ventilation (for outcome ventilator-associated pneumonia).

For the primary and secondary objectives, advanced survival techniques (competing risks and multistate models) will be applied. Discharge and death will be considered as competing events for ICU pneumonia. Adapted Cox regression models will be applied for each 
event separately as well as for the sub-distribution of ICU pneumonia. The clustering of the data (readmission, patients within ICU, country) will be acknowledged using shared frailty methodology, stratification or robust variance. The time-dependency of cumulative hazards and incidences will be graphically displayed, by risk factors of interest. Hazard ratios with $95 \%$ CI will be calculated univariately and selected for the multivariate model using an established Akaike's information criterion for model selection. A risk prediction model will be developed to quantify the risk for acquiring $S$. aureus or $P$. aeruginosa ICU pneumonia during ICU stay by using a composite score of independent risk factors.

\section{Quality assurance}

Data will be entered in a web-based electronic data capture system that was designed for ASPIRE-ICU. The study site will enter data in the electronic data capture system from the subject's source documents (i.e. medical chart). Information linking the subject ID to the subject's medical file (only applicable for study cohort subjects) will be kept in a secure place at the participating study site.

Monitoring will include 100\% source data verification for the first three enrolled study cohort subjects at each site, and then $10 \%$ of the remaining enrolled study cohort subjects.

\section{Discussion}

This manuscript describes the objectives and design of ASPIRE-ICU, an observational cohort study addressing risk factors for $S$. aureus and $P$. aeruginosa ICU pneumonia. Certain choices have been made in the design that warrant mention and further discussion.

\section{Definition of pathogen-specific pneumonia}

The definition of $S$. aureus or $P$. aeruginosa ICU pneumonia is based on pre-defined pneumonia criteria in combination with the presence of the bacterium in an appropriate sample around the time of diagnosis, which are aligned with endpoints being used for two randomized controlled trials for prevention of $S$. aureus and $P$. aeruginosa pneumonia [8]. Thus, in case of cultures that yield multiple possible pathogens, it may be that the diagnosis of $S$. aureus or $P$. aeruginosa pneumonia is made incorrectly. The distinction between colonization and infection is sometimes difficult to assess. There is however no 'reference standard' to reliably assess the causative pathogen [3]. We have contemplated quantitative cultures in all pneumonia patients, but it was not feasible to implement this at each site. Quantitative measurements will however be applied on study samples received by the central laboratory, and thus will provide additional retrospective information. Furthermore, other outcomes, such as mortality will provide objective outcome information in addition to a diagnosis of pneumonia.

\section{Enrichment strategy}

In this study, $50 \%$ of the study cohort population is $S$. aureus colonized at ICU admission to 'enrich' the study population with $S$. aureus carriers (in nose or lower respiratory tract), while this naturally occurs in approximately $20-25 \%$ of the ICU population [10]. This was chosen as $S$. aureus carriage is being studied as one of the main known risk factors for subsequent $S$. aureus disease, thus without enrichment, the population needed for equal precision would be much larger $[9,11,12]$. However, the limitation of this choice is that one can argue that a population as such is not representative of the general ICU population, thus incidence estimation as well as assessing risk factors for $P$. aeruginosa ICU pneumonia may be suboptimal. We acknowledge this, and for this reason the surveillance population was included. Their data will allow distribution of baseline factors with the study cohort in an effort to assess the ubiquity of results across both groups. Furthermore, it can serve to identify potential bias between participants and non-participants. Considering that $P$. aeruginosa colonization at ICU admission is relatively rare and that colonization often occurs after ICU admission, no recruitment selection for $P$. aeruginosa colonized subjects will take place [13].

\section{Routine surveillance}

This study utilizes routine $S$. aureus screening conducted at each participating site as the basis for eligibility assessment. For consistency of screening results across sites, all swabs will be plated under protocol on the same chromogenic agar plates (Colorex agar, bioTRADING Benelux) provided by the ASPIRE-ICU study team. In the original protocol, a nose swab and ETA sample (sputum sample if non-intubated) are collected at screening. However, soon after study start, the protocol was amended to include a throat sample as a LRT sample, in case ETA and sputum were both not feasible.

\section{Future perspectives}

In this era of increasing antimicrobial resistance, the research field is steadily exploring other therapies, for example prophylactic therapies such as antibody-based preventive measures. A potential advantage of monoclonal antibodies (mAbs) is that they will not encourage bacterial resistance to the same extent as antibiotics, and may even augment antibiotic effectiveness [14]. This study was designed in part to provide crucial information on the incidence, patient-related and contextual factors of ICU pneumonia caused by $S$. aureus and $P$. aeruginosa, but also inform the design of future phase 
III trials, that will investigate mAbs effectiveness against $S$. aureus and $P$. aeruginosa. Two large COMBACTE phase II trials, SAATELLITE (A Human Monoclonal Antibody Against Staphylococcus aureus Alpha Toxin in Mechanically Ventilated Adult Subjects) and EVADE (Effort to Prevent Nosocomial Pneumonia Caused by Pseudomonas aeruginosa in Mechanically Ventilated Subjects) have already started [8]. SAATELLITE investigates the effect of MEDI4893, a mAb targeting S. aureus alpha toxin and EVADE studies MEDI3902, which is another mAb that simultaneously targets PcrV and Psl on the surface of the $P$. aeruginosa bacterium, in subjects at risk for ICU pneumonia $[5,6,8]$. These targeted therapies, if proven effective, may be used in the future for patients at highest risk. This study will help to identify the subset of patients that will likely benefit most.

\section{Conclusion}

This epidemiological cohort study on $S$. aureus and $P$. aeruginosa ICU pneumonia aims to add significant information to the literature on predictors for this event. This will help refine the design of Phase III trials and may benefit patients at risk for nosocomial infections, by providing protective measures.

\section{Additional file}

Additional file 1: S.01. Complete list of objectives and endpoints. Table S 02. Schedule of procedures. S.03. Complete definition of study endpoints. (DOCX 29 kb)

\section{Abbreviations}

ASPIRE-ICU: Advanced understanding of Staphylococcus aureus and Pseudomonas aeruginosa Infections in EuRopE; Cl: Confidence interval; COMBACTE: COMBatting AntibiotiC resisTance in Europe; ETA: Endotracheal aspirate; ICU: Intensive Care Unit; LOS: Length of stay; LRT: Lower respiratory tract; $\mathrm{mAb}$ : Lower respiratory tract; $P$. aeruginosa: Pseudomonas aeruginosa; $S$. aureus: Staphylococcus aureus

\section{Acknowledgments}

We would like to thank the following people for their contributions while writing this study protocol: Stephan Harbarth, Mark Eickhoff, Bruno François, Frank Coenjaerts, Uwe Völker, Barbara Bröker, Andreas Peschel, Willem Van Wamel, Gerard Lina, Jos Van Strijp, Samir Kumar-Singh, Antonio Oliver, Christian Van Delden, Thilo Kohler, Tom van der Poll, Craig Maclean.

\section{Funding}

This research project receives support from the Innovative Medicines Initiative Joint Undertaking under grant agreement no. 115523 and 115737 resources which are composed of financial contribution from the European Union Seventh Framework Programme (FP7/2007-2013) and EFPIA companies in kind contribution.

\section{Availability of data and materials}

The datasets generated and analyzed during the current study are not publicly available due to confidentiality reasons but are available from the corresponding author upon scientific review and approval of the request by the Study's Scientific Committee. A summary of the results will be published on clinicaltrials.gov.

\section{Authors' contributions}

FPP authored the original study protocol and this manuscript. FPP, DPRT, FS, $C L, R K, D P, L T, H G, S M K, M J M B$ and JAJW together created the study design. MW and SW, with help of all other authors designed the Statistical Analysis Plan. All authors read and approved the study protocol and the final manuscript.

\section{Ethics approval and consent to participate}

This study will be conducted according to the principles of the Declaration of Helsinki, in accordance with the Medical Research Involving Human Subjects Act (WMO) and local guidelines in the participating countries. [15] Central IEC's in each participating country gave a favorable opinion to the study protocol, including informed consent procedures.

\section{Consent for publication}

Not applicable.

\section{Competing interests}

FS is an employee of AstraZeneca. RK is an employee of Medlmmune. The other authors declare that they have no competing interests.

\section{Publisher's Note}

Springer Nature remains neutral with regard to jurisdictional claims in published maps and institutional affiliations.

\section{Author details}

${ }^{1}$ Julius Center for Health Sciences and Primary Care, University Medical Center Utrecht, HP Stratenum 6.131, PO Box 85500, 3508 GA Utrecht, The Netherlands. ${ }^{2}$ Institute for Medical Biometry and Statistics, Faculty of Medicine and Medical Center, University of Freiburg, Freiburg, Germany. ${ }^{3}$ Medlmmune, Gaithersburg, MD, USA. "Laboratory of Medical Microbiology, Vaccine \& Infectious Disease Institute (VAXINFECTIO), University of Antwerp, Wilrijk, Belgium. ${ }^{5}$ AstraZeneca Pharmaceuticals LP, Gaithersburg, MD, USA. ${ }^{6}$ Department of Medical Microbiology, University Medical Center Utrecht, Utrecht, Netherlands. ${ }^{7}$ Amphia Hospital, Breda, The Netherlands.

Received: 17 August 2017 Accepted: 14 September 2017 Published online: 25 September 2017

\section{References}

1. Chastre J, Fagon J-Y. Ventilator-associated pneumonia. Am J Respir Crit Care Med American Thoracic SocietyNew York, NY. 2002;165:867-903. doi:10.1164/airccm.165.7.2105078.

2. Vincent JL, Bihari DJ, Suter PM, Bruining HA, White J, Nicolas-Chanoin MH, et al. The prevalence of nosocomial infection in intensive care units in Europe. Results of the European prevalence of infection in intensive care (EPIC) study. EPIC international advisory committee. JAMA. 1995;274:639-44. Available: http://www.ncbi.nlm.nih.gov/pubmed/7637145.

3. Douglas IS. New diagnostic methods for pneumonia in the ICU. Curr Opin Infect Dis. 2016;29:197-204. doi:10.1097/QCO.0000000000000249.

4. François B, Jafri HS, Bonten M. Alternatives to antibiotics. Intensive Care Med. 2016;42:2034-6. doi:10.1007/s00134-016-4339-y.

5. Hua L, Hilliard JJ, Shi Y, Tkaczyk C, Cheng LI, Yu X, et al. Assessment of an anti-alpha-toxin monoclonal antibody for prevention and treatment of Staphylococcus Aureus-induced pneumonia. Antimicrob agents Chemother. Am Soc Microbiol (ASM). 2014;58:1108-17. doi:10.1128/AAC.02190-13.

6. DiGiandomenico A, Warrener P, Hamilton M, Guillard S, Ravn P, Minter R, et al. Identification of broadly protective human antibodies to Pseudomonas Aeruginosa exopolysaccharide Psl by phenotypic screening. J Exp Med. 2012;209:1273-87. doi:10.1084/jem.20120033.

7. Kostyanev T, Bonten MJM, O'Brien S, Steel H, Ross S, François B, et al. The innovative medicines Initiative's new drugs for bad bugs programme: European public-private partnerships for the development of new strategies to tackle antibiotic resistance. J Antimicrob Chemother Oxford University Press. 2016;71:290-5. doi:10.1093/jac/dkv339.

8. François B, Chastre J, Eggiman P, Laterre P-F, Torres A, Sanchez M, et al. The SAATELLITE and EVADE clinical studies within the COMBACTE consortium: a public-private collaborative effort in designing and performing clinical trials for novel antibacterial drugs to prevent Nosocomial pneumonia: table 1. Clin Infect Dis. 2016;63:S46-51. doi:10.1093/cid/ciw245. 
9. Paling FP, Wolkewitz M, LGM B, PMC KK, DSY O, Depuydt P, et al. Staphylococcus Aureus colonization at ICU admission as a risk factor for developing S. Aureus ICU pneumonia. Clin Microbiol Infect. 2017;23:49.e949.e14. doi:10.1016/..cmi.2016.09.022.

10. Wertheim HFL, Melles DC, Vos MC, van Leeuwen W, van Belkum A, Verbrugh HA, et al. The role of nasal carriage in Staphylococcus Aureus infections. Lancet Infect Dis. 2005;5:751-62. doi:10.1016/S14733099(05)70295-4.

11. Ziakas PD, Anagnostou T, Mylonakis E. The prevalence and significance of methicillin-resistant Staphylococcus Aureus colonization at admission in the general ICU setting: a meta-analysis of published studies. Crit Care Med. 2014;42:433-44. doi:10.1097/CCM.0b013e3182a66bb8.

12. Davis KA, Stewart JJ, Crouch HK, Florez CE, Hospenthal DR. Methicillinresistant Staphylococcus Aureus (MRSA) nares colonization at hospital admission and its effect on subsequent MRSA infection. Clin Infect Dis. 2004;39:776-82. doi:10.1086/422997.

13. Kollef MH, Chastre J, Fagon J-Y, François B, Niederman MS, Rello J, et al. Global prospective epidemiologic and surveillance study of ventilatorassociated pneumonia due to Pseudomonas Aeruginosa. Crit Care Med. 2014;42:2178-87. doi:10.1097/CCM.0000000000000510.

14. Saylor C, Dadachova E, Casadevall A. Monoclonal antibody-based therapies for microbial diseases. Vaccine. 2009;27(Suppl 6):G38-46. doi:10.1016/j. vaccine.2009.09.105.

15. World Medical Association. World medical association declaration of Helsinki: ethical principles for medical research involving human subjects. JAMA. 2013;310:2191-4. doi:10.1001/jama.2013.281053.

\section{Submit your next manuscript to BioMed Central and we will help you at every step:}

- We accept pre-submission inquiries

- Our selector tool helps you to find the most relevant journal

- We provide round the clock customer support

- Convenient online submission

- Thorough peer review

- Inclusion in PubMed and all major indexing services

- Maximum visibility for your research

Submit your manuscript at www.biomedcentral.com/submit

C) Biomed Central 